\title{
A case of immune thrombocytopenic purpura complicated by HELLP syndrome
}

\author{
S Prithipal, MB ChB, Dip Obst (SA), FCOG (SA) \\ Department of Obstetrics and Gynaecology, Nelson R Mandela School of Medicine, University of KwaZulu-Natal, Durban, South Africa
}

Corresponding author: S Prithipal (sudhir.prithipal@kznhealth.gov.za)

The combination of immune thrombocytopenic purpura and haemolysis, elevated liver enzymes and low platelets (HELLP) syndrome is rare, with only two previous case reports published. Management of the two conditions is vastly different, and the diagnosis of HELLP syndrome superimposed on immune thrombocytopenic purpura may be difficult, resulting in delayed management.

S Afr J Obs Gynae 2019;25(1):4-5. DOI:10.7196/SAJOG.2019.v25i1.1353

A 35-year-old female, gravida 2, para 1, at 30 weeks' gestation, presented to a South African (SA) regional-level hospital with a frontal headache of 1 day's duration. There were no other symptoms of imminent eclampsia. She was a foreign national and had received previous antenatal care at a different centre in SA. She was diagnosed with chronic hypertension privately at booking (7 weeks' gestation), and put onto methyldopa. Her HIV test was negative. At 23 weeks' gestation, blood tests revealed a moderate thrombocytopenia with a platelet (PLT) count of $63 \times 10^{9} / \mathrm{L}$. Her blood pressure (BP) was controlled, and all other blood tests were essentially normal. She was diagnosed as having immune thrombocytopenic purpura (ITP), and commenced on prednisone (defaulted treatment).

On arrival at the regional hospital at 30 weeks' gestation, her BP was $188 / 96 \mathrm{mmHg}$, with $2+$ proteinuria on urinary dipstick. Repeat $\mathrm{BP}$ was $180 / 113 \mathrm{mmHg}$, and she was given a stat dose of nifedipine and commenced on magnesium sulphate. She was transferred to high care in the labour ward (LWHC). Blood test results showed a haemoglobin $(\mathrm{Hb})$ of $11.8 \mathrm{~g} / \mathrm{dL}$ and a platelet count of $9 \times 10^{9} / \mathrm{L}$. Alanine transaminase (ALT) and lactate dehydrogenase (LDH) were $47 \mathrm{IU} / \mathrm{L}$ and $333 \mathrm{IU} / \mathrm{L}$, respectively. The patient was transfused with two pooled platelets and recommenced on prednisone. Betamethasone was administered for fetal lung maturity. The patient's BP was controlled. The initial plan of management was to be conservative with regard to the pregnancy, and to optimise the maternal condition.

The patient's BP was well controlled over the next 48 hours. However, her blood test results deteriorated (Table 1).

The rising ALT and LDH combined with the decline in the $\mathrm{Hb}$ suggested that the patient was developing haemolysis, elevated liver enzymes and low platelets (HELLP) syndrome. The case was discussed with the anaesthetist, and arrangements made to deliver the patient via caesarean section (CS). Under platelet cover, a CS was performed, and a live female infant with good Apgar scores delivered. The patient was transferred back to LWHC, and then to the postnatal ward as a boarder mother, as her baby had a prolonged neonatal intensive care admission. By day 5 postoperatively, her platelet count had improved to $182 \times 10^{9} / \mathrm{L}$, and her other blood parameters were improving.

\section{Table 1. Blood test results}

\begin{tabular}{llll}
\hline Parameter & Admission & Day 1 & Day 2 \\
\hline $\mathrm{Hb}(\mathrm{g} / \mathrm{dL})$ & 11.8 & 10.7 & 10.6 \\
$\mathrm{PLT}\left(\times 10^{9} / \mathrm{L}\right)$ & 9 & 47 & 58 \\
$\mathrm{ALT}(\mathrm{IU} / \mathrm{L})$ & 47 & 50 & 156 \\
$\mathrm{LDH}(\mathrm{IU} / \mathrm{L})$ & 333 & 333 & 426 \\
$\begin{array}{l}\mathrm{Hb}=\text { haemoglobin; } \\
\text { dehydrogenase. }\end{array}$ &
\end{tabular}

The case was discussed with the departments of internal medicine and haematology, and the patient continued on prednisone at a dose of $1 \mathrm{mg} / \mathrm{kg} /$ day. Post delivery, the patient revealed that she had been diagnosed with ITP in 2013 and had started on prednisone therapy. She had returned to her home country for a year in 2015, and defaulted treatment and follow-up. The importance of compliance was stressed to the patient, and after a 56-day stay in the nursery, the baby was discharged. The patient was discharged on maintenance steroids, and follow-up was arranged with the haematology department.

\section{Discussion}

ITP as a cause for thrombocytopenia (TCP) in pregnancy is rare, with an incidence of $<10$ per 10000 pregnancies. ${ }^{[1]}$ The diagnosis of ITP outside pregnancy is one of exclusion. Standard treatment of ITP usually commences with a corticosteroid such as prednisone. The dose is determined according to the patient's weight and response to therapy, based on an improvement in the PLT count. HELLP syndrome is regarded as a form of severe pre-eclampsia. There have been two main classification systems, the Tennessee and Mississippi. There is also a concept of partial HELLP, without haemolysis as a prerequisite, or lower levels of $\mathrm{LDH}$ required for $\operatorname{diagnosis.~}^{[2]}$

The patient in our case already had severe TCP owing to her ITP. Other initial parameters were not wholly indicative of HELLP. This led to a slight delay in diagnosis until her ALT levels became markedly raised.

HELLP syndrome in a patient with ITP is extremely rare, with only two other case reports found during a literature review. ${ }^{[3,4]}$ There is no known association between ITP and pre-eclampsia. The 
severity of the TCP in ITP may confound the diagnosis of HELLP syndrome, and result in delays of appropriate management. As this is a form of severe pre-eclampsia, a major concern in a patient with severe TCP is intracranial haemorrhage. Stringent BP control in a high-care setting, with stabilisation of the mother, is a priority.

Patients with ITP who develop pre-eclampsia must be monitored carefully for the development of features of severe pre-eclampsia. This would include a thorough history, physical examination and, most importantly, relevant laboratory investigations (ALT, LDH, renal function). Vigilance is required for the detection of other complications of severe pre-eclampsia (e.g. pulmonary oedema), and a plan for delivery may be required, as this is the only cure. If gestational age is $<34$ weeks, up to 48 hours may be needed for corticosteroid therapy. ${ }^{[2]}$ HELLP syndrome remote from term is not usually managed expectantly beyond 48 hours, although individualisation of cases may be necessary where the gestational age is of borderline viability. The PLT count usually decreases slightly immediately post delivery, before improving and returning towards normal within 7 days.

ITP during pregnancy must be managed by a multidisciplinary team to ensure holistic and optimal care of the patient. The mainstay of treatment is corticosteroids or intravenous immunoglobulin (IVIg). ${ }^{[5]}$ IVIg is the more expensive treatment, and used if a rapid increase in PLT count is required, e.g. close to delivery. Other immunosuppressive therapies for ITP are contraindicated in pregnancy, and splenectomy is a last resort if medical treatment fails. Ideally, splenectomy should be performed either pre or post pregnancy. The main aim of treatment is to ensure that the patient does not have a risk of spontaneous bleeding, as such episodes (especially intracranial bleeding) may be fatal. It is not the aim to achieve a normal PLT count. If a patient is symptom-free and her PLT count is $\geq 20 \times 10^{9} / \mathrm{L}$, therapy is usually deferred until delivery is imminent. ${ }^{[5]}$ Patients with PLT counts $<20 \times 10^{9} / \mathrm{L}$ require therapy irrespective of symptoms, to reduce complications of bleeding. For a normal vaginal delivery, a PLT count of $>50 \times 10^{\%} / \mathrm{L}$ is regarded as safe, while a PLT count of $>80 \times 10^{9} / \mathrm{L}$ is required for epidural, spinal anaesthesia or CS. ${ }^{[5]}$ Post delivery the PLT count must be monitored, and patients must be observed closely for postpartum haemorrhage. Follow-up with the haematologist is mandatory.

There are no clear guidelines regarding management of a patient with ITP complicated by HELLP syndrome. Maternal wellbeing takes precedence. Optimisation of BP, monitoring for other complications (e.g. bleeding) followed by delivery of the patient should be key. Ideally, these patients should deliver via the vaginal route, to reduce the chance of postpartum haemorrhage and prevent complications of bleeding at CS. Combined management with a haematologist is of vital importance throughout the pregnancy and afterwards.

In the case presented, defaulting medication led to severe TCP, and placed this patient at risk of several complications, especially due to her markedly raised BP on admission. She displayed a good response to the corticosteroids, and emphasis was placed on the importance of compliance and follow-up upon her discharge from hospital.

Acknowledgements. None.

Author contributions. Sole author.

Funding. None.

Conflicts of interest. None.

1. Bergmann F, Rath W. The differential diagnosis of thrombocytopenia in pregnancy. Dtsch Arztebl Int 2015;112(47):795-802. https://doi.org/10.3238/arztebl.2015.0795

2. Haram K, Svendsen E, Abildgaard U. The HELLP syndrome: Clinical issues and management. A review. BMC Pregnancy Childbirth 2009;9(8):1-15. https://doi.org/10.1186/1471-2393-9-8

3. Ben S, Rodríguez F, Severo C, Debat N. A case of HELLP syndrome in a patient with immune thrombocytopenic purpura. Obstet Gynecol Int 2010;1:692163. https://doi. org/10.1155/2010/692163

4. Mehta T, Parikh GP, Shah VR. Triad of idiopathic thrombocytopenic purpura, preeclampsia, and HELLP syndrome in a parturient: A rare confrontation to the anesthetist. Case Rep Anesthesiol 2014;1:139694. https://doi.org/10.1155/2014/139694

5. British Committee for Standards in Haematology General Haematology Task Force. Guidelines children and in pregnancy. Br J Haematol 2003;120(4):574-596. https://doi.org/10.1046/j.13652141.2003.04131.x

Accepted 11 June 2019 